\title{
Ocular Fundus Imaging: From Structure to Function
}

\author{
P. Serranho ${ }^{1, *}$, C.Maduro ${ }^{1,2}$, T.Santos ${ }^{1,2}$, \\ J.Cunha-Vaz ${ }^{1,2}$, R.Bernardes ${ }^{1,2}$ \\ ${ }^{1}$ IBILI-Institute of Biomedical Research on Light and Image \\ Faculty of Medicine, University of Coimbra \\ ${ }^{2}$ AIBILI - Association for Innovation and Biomedical \\ Research on Light and Image \\ Coimbra, Portugal \\ *pserranho@fmed.uc.pt
}

\author{
Adérito Araújo ${ }^{3}$, Silvia Barbeiro ${ }^{3}$ \\ ${ }^{3}$ Mathematics Department \\ Faculty of Science and Technology, University of Coimbra \\ Coimbra, Portugal
}

\begin{abstract}
Imaging the ocular fundus, namely the retina, to detect and/or monitor changes over time from the healthy condition is of fundamental importance to assess onset and disease progression and is a valuable tool to understand the basic mechanisms of ocular diseases. Current trends point to the need for less or non-invasive approaches, to the need for detailed (higher spatial and temporal resolution) imaging systems and to the quantification as opposed to qualitative classification of any findings.

In this work we present a snapshot of our research by presenting two examples of technical development aiming to obtain structural and function information from the human retina, in vivo, using non-invasive techniques, namely optical coherence tomography imaging. Based on our experience and developed work, we are now starting to bridge the gap to brain imaging as the eye is only the starting point of vision.
\end{abstract}

Index Terms-Optical Coherence Tomography, despeckling, image segmentation.

\section{INTRODUCTION}

Vision is one of the most valued senses in humans and its loss or degradation is therefore of paramount importance. The eye, where it all starts, plays in this way a fundamental role in vision by representing the front-end part of a complex and still not fully understood system.

Within our research group we are focused in the posterior segment of the human eye, the retina, and in developing procedures/imaging modalities able to provide early quantitative indications on any alterations occurring in the ocular fundus. These alterations can be either structural, functional or both and we aim to understand how they correlate with each other and with disease onset/progression, eg. diabetes, one of the main causes of vision loss in developed countries.

Moreover, we aim to move from qualitative to quantitative imaging, from invasive to non-invasive systems and from documentation to diagnosis imaging.

In this way, we have been actively contributing to knowledge in field of diabetic retinopathy, mainly, by developing functional imaging modalities, unifying different sources into multimodal imaging and quantifying traditional imaging modalities.
We are currently deeply involved in getting functional information from structural imaging modalities as the wide spread optical coherence tomography (OCT) imaging modality.

In the rest of this paper we will briefly step by recent contributions in relation with the OCT, namely the development of a complex diffusion despeckling filter and work on automated segmentation of retinal structures, fundamental steps towards functional imaging through OCT.

OCT is a non-invasive imaging modality with an increasing number of applications and it is becoming an essential tool in ophthalmology [1] allowing in vivo highresolution cross-sectional imaging of the retinal tissue. However, as any imaging technique that bases its image formation on coherent waves, OCT images suffer from speckle noise, which reduces its quality.

Speckle noise creates a grainy appearance that can mask diagnostically significant image features (small or low reflectivity features) and reduce the accuracy of segmentation and pattern recognition algorithms [2-5].

In this work we present some recent developments to overcome these problems. We suggest an improved adaptive complex diffusion despeckling filter that suppresses speckle noise while preserving features within the tissue. This can be seen as a first step towards the segmentation of the image.

We also present the foundations of a fully automatic computational light support vector machine (SVM) based segmentation algorithm for segmentation of the retina in an OCT image. The classification for the training set is obtained by gradient methods, namely the mean-shift algorithm. No manual input is needed, opposite to other segmentation methods based on classification [6,7].

\section{METHODS}

\section{A. Improved adaptive complex diffusion despeckling filter}

The general nonlinear complex diffusion filtering (NCDF) method $[4,5]$ looks for the solution of the complex diffusion equation 


$$
\frac{\partial I}{\partial t}=\nabla \cdot(D \nabla I),
$$

where $\nabla \cdot$ is the divergence, $\nabla$ is the gradient and $\mathrm{D}$ is the diffusion coefficient, where $D$ is chosen in order to preserve edges, that is, dependent on the gradient or laplacian of the image. Gilboa et al. [5] showed that to prevent from computing derivatives of the image that become highly instable with noise, complex diffusion should be used. The method was designed in a way that, as iterations go by, the imaginary part of the image retrieves feature and edge information of a filtered version of the original image, as showed in [5]. The adequate coefficient of diffusion also used by Salinas et al. [4] for OCT is given by

$$
D=\frac{\exp (i \theta)}{1+(\operatorname{Im}(I) / \kappa \theta)^{2}},
$$

where $i=\sqrt{-1}, \kappa$ a threshold parameter, $\mathrm{d}$ a phase angle close to zero and I the image. Equation (1) was then discretized by a forward in time centered in space finite difference scheme that was applied with a fixed time step.

We suggest two main alterations to the method [8], regarding the parameter $\kappa$ and an adaptive time step.

The choice for the parameter $\kappa$ (Eq. 2) is important, as it modulates the spread of the diffusion coefficient in the vicinity of its maximum, that is, at edges and homogeneous areas, where the image laplacian vanishes. In this way a choice of $\kappa$ that is more conservative within the retinal tissue (high intensities) and less conservative outside the retina (low intensities) is advised, giving rise to

$$
\kappa=\kappa_{M A X}+\left(\kappa_{M I N}-\kappa_{M A X}\right) \frac{g-\min (g)}{\max (g)-\min (g)},
$$

where $\min (\mathrm{g})$ and $\max (\mathrm{g})$ stand for the minimum and maximum of $g$, respectively, with $g$ being defined as

$$
g=G_{N, \sigma} * \operatorname{Re}(I),
$$

where $*$ is the convolution operator and $G_{N, \sigma}$ is a local average (gaussian) kernel of size $\mathrm{NxN}$ and standard deviation $\mathrm{u}$.

Since the coefficient of diffusion depends on the imaginary part of the image (Eq. 2), which is closely related with its gradient, and, due to noise, this gradient is much higher in the initial steps of the diffusion process, we propose to consider an adaptive time step, as opposed to the majority of nonlinear complex diffusion processes that adopt a constant one. In this way, we consider

$$
\Delta t^{(n)}=\frac{1}{\alpha}\left[a+b \exp \left\{-\max \left(\left|\operatorname{Re}\left(\partial I^{(n)} / \partial t\right)\right| / \operatorname{Re}\left(I^{(n)}\right)\right)\right\}\right]
$$

where $\left|\operatorname{Re}\left(\partial I^{(n)} / \partial t\right)\right| / \operatorname{Re}\left(I^{(n)}\right)$ is the fraction of change of the image/volume at iteration $n, \breve{U}=4$ in $2 \mathrm{D}$ images and parameters $a$ and $b$ control the time step (with $a+b \mathrm{O} \mathbf{1}$ ). This choice satisfies the stability condition for this method [9].
The typical evolution of $\Delta t^{(n)}$ over the iterative process is shown in figure 1.

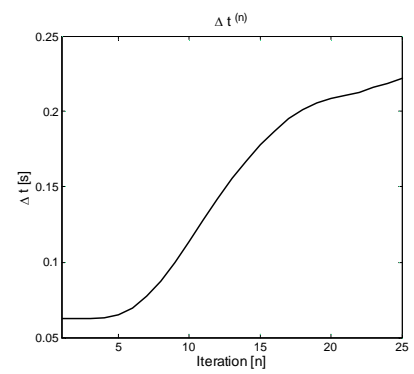

Fig. 1 i Typical evolution of the time step

(q) for the proposed adaptive process.

As expected, a small step size is used at the initial iterations in which higher values of $D$ can be found due to the speckle noise. The step size increases as iterations goes by, that is, as speckle is being suppressed.

With appropriate changes this method can be applied in 3D [8], which has application if one wants to process the whole OCT volume data.

\section{B. Segmentation of the Retina in OCT images}

Filtered images as described in the previous section can be used for segmentation purposes. Several segmentation methods have been used in OCT, such as gradient $[10,11]$ and support vector machines (SVM) based methods [6,7] among others. While gradient methods usually do not work in areas of low quality signal, SVM methods require a previously segmented training set. In this way, we propose to use gradient based methods in areas where the quality of segmentation is validated by an automatic criterion as a training set for a SVM method.

The method consists of several steps. First, one divides the fundus image in nine areas, disposed in a $3 \times 3$ setting. In each of these areas, we search by an A-scan that can be correctly segmented by gradient based methods, namely using the meanshift algorithm. To validate the correctness of the segmentation, we considered a specific automatic criterion that is based on the statistics of the signal in each segmented region. At the end of this first step, we have nine segmented A-scans that can be used as training data for an SVM algorithm to classify the whole volume.

As features for the SVM method we consider the intensity and its logarithm, as well as the mean intensity in windows of different scales around, above and below the pixel and the variance of the intensity in windows of different scales, in a total of 14 features. Moreover, we consider a radial basis function kernel and a one-against-all comparison strategy for the SVM classifier.

In this way one can obtain a fully automatic segmentation 'of four regions within an OCT image: the vitreous, the area between the inner limiting membrane and the outer segment layer, the area containing the outer segment layer and the retinal pigment epithelium and, finally, the choroid. We stress that there is no need of a manual segmentation to establish a training set and that the number of used features is small considering published literature on this field $[6,7]$. 


\section{RESULTS}

\section{A. Improved adaptive complex diffusion despeckling filter}

Tests were conducted in the real human eye fundus OCT data using the the high-definition spectral domain Cirrus OCT (Carl Zeiss Meditec, Dublin, CA, USA). We considered 32 B-scans using the macular cube protocol from 32 eye fundus scans from 13 healthy volunteers, 3 eyes with choroidal neovascularization, 2 with cystoid macular edema, 9 with diabetic retinopathy and 5 with age-related macular degeneration.

The results of the despeckling method can be seen in figures 2 and 3 where the traditional and improved complex diffusion methods are compared. In figure 2 we show results for a synthetic profile with an abrupt and a ramp edge. In figure 3 we show the application to an OCT image.

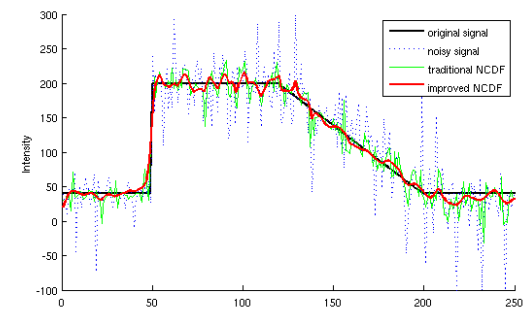

Fig. 2 ï Comparison of the original (black) and noisy (dotted blue) profile with the traditional (green) and improved (red) NCDF methods.
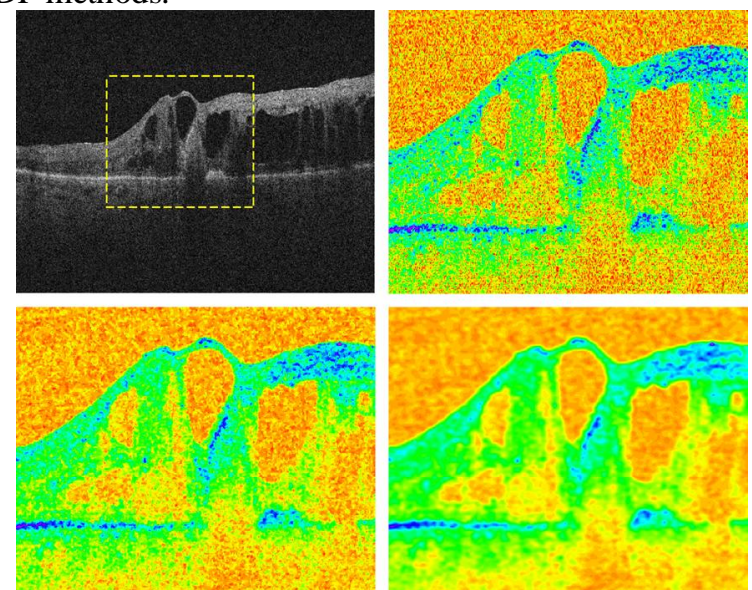

Fig. 3 ï Original high resolution OCT scan (top left), region of interest in color code (top right) and traditional (bottom left) and improved (bottom right) NCDF filtered images.

Both images show that the improved version has better performance, both in edge and feature preservation. The following table obtained for a synthetic image to which was added random noise in 50 different runs also shows the significantly better performance of the improved NCDF.
Table 1. Performance metrics computed from 50 runs (mean \pm SD). LI = lowintensity areas; $\mathrm{HI}=$ high-intensity areas; $\mathrm{MSE}=$ mean square error; $\mathrm{ENL}=$ effective number of looks; $\mathrm{CNR}=$ contrast to noise ratio.

\begin{tabular}{lccc}
\hline & Noise & $\begin{array}{c}\text { Traditional } \\
\text { NCDF }\end{array}$ & $\begin{array}{c}\text { Improved } \\
\text { NCDF }\end{array}$ \\
\hline $\mathrm{ENL}_{\mathrm{LI}}$ & $1.0 \pm 0.1$ & $4.6 \pm 0.3$ & $46.3 \pm 7.5$ \\
\hline $\mathrm{MSE}_{\mathrm{LI}}$ & $1604.3 \pm 85.1$ & $348.4 \pm 22.2$ & $37.1 \pm 6.0$ \\
\hline $\mathrm{ENL}_{\mathrm{HI}}$ & $24.9 \pm 1.4$ & $115.3 \pm 7.1$ & $409.2 \pm 51.3$ \\
\hline $\mathrm{MSE}_{\mathrm{HI}}$ & $1607.7 \pm 92.3$ & $348.6 \pm 21.6$ & $99.9 \pm 11.9$ \\
\hline $\mathrm{CNR}$ & $2.8 \pm 0.1$ & $6.1 \pm 0.1$ & $13.8 \pm 0.7$ \\
\hline
\end{tabular}

The computing time for the synthetic image data used in the table (with a diffusion time of 12 seconds for the traditional NCDF) is (mean $\pm \mathrm{SD}$ ) $4.2 \pm 0.05$ seconds for the traditional NCDF. The proposed formulation (with a diffusion time of 3 seconds), however, presents a computing time $5.6 \pm 0.08$ seconds, showing the computational cost of the adaptive step.

However, for real data our method performs faster. In fact, we got comparative performances of $3.4 \pm 0.06$ to $2.1 \pm 0.09$ seconds, respectively, for the traditional and proposed filter formulation (61.8\% of computing time).

If instead of using a fixed diffusion time one uses as stopping criteria a given level of ENL, our improved method also performs better. To achieve the same ENL over a vitreous region as the traditional NCDF filter (12 seconds of diffusion time) for the above test set (32 B-scans) the comparative performance is $3.4 \pm 0.06$ and $1.1 \pm 0.12$ seconds, respectively, for the traditional and the proposed formulation, i.e., the same results were achieved in $34 \%$ of the computing time.

\section{B. Segmentation of the Retina in OCT images}

As previously mentioned, we divide the fundus reference image into nine areas displayed in a $3 \times 3$ setting. In each area we look for a valid segmented A-scan by gradient methods as illustrated in figure 4 and take this set as training set for the SVM algorithm.

The final segmentation obtained by the SVM algorithm for the $60^{\text {th }} \mathrm{B}$-scan is illustrated in figure 5. In a qualitative evaluation the accuracy of the segmentation is similar across the whole volume for OCT data of 4 healthy eyes, though no quantitative performance metrics were still computed. For healthy eyes, the areas of lower accuracy are mainly located at the fovea and below blood vessels, due to the associated shadows. 

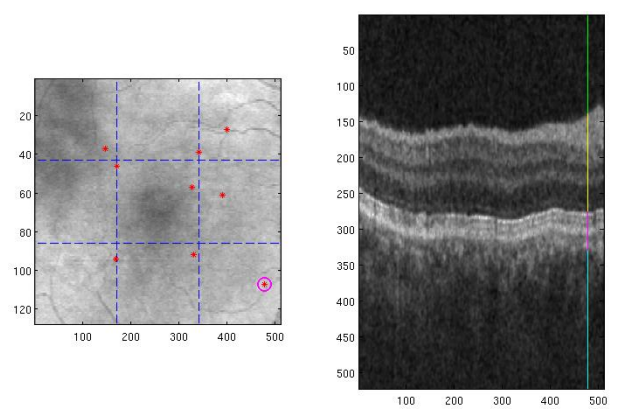

Fig. 4 ï Chosen A-scans (red star) in each $3 \times 3$ divided areas of the fundus image (left) and the respective A-scan segmentation (right) obtained by gradient methods for the circled A-scan on the left.

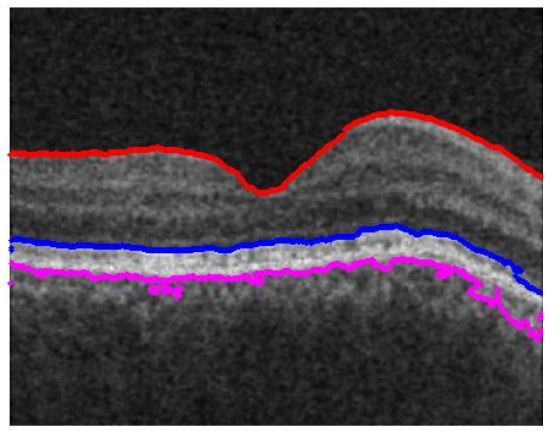

Fig. 5 ï SVM segmentation of the inner limiting membrane (red), upper outer segment layer (blue) and upper choroid (pink).

\section{DISCUSSION AND CONCLUSIONS}

In this report, we propose a new formulation of the nonlinear complex diffusion filter and demonstrate its advantages with regard to edge preservation, speckle filtering capabilities and potential to recover the original (uncorrupted) signal, hence the use of synthetic data.

Based on our results the proposed formulation achieved a superior performance while retaining all the advantages of nonlinear complex diffusion filters. One particular advantage to the original formulation is that the $ə$ parameter value does not need to be defined beforehand; instead, it adapts itself to the data.
As a proof of concept, we also illustrated how gradienttype methods can be used for establishing a training set for SVM segmentation algorithms. This allows one to obtain a fully automatic segmentation procedure for OCT retinal images.

Current research includes an active contour approach as pos-processing to increase the accuracy of the segmentation, as well as dealing with blood vessel shadows that induce errors in the segmentation. Future work will also address pathologic cases, namely segmentation in OCT data with edema due to Diabetic Retinopathy.

\section{REFERENCES}

[1] W. Drexler, U. Morgner, R. K. Ghanta, F. X. Kartner, J. S. Schuman and J. G. Fujimoto, ñUltrahigh-resolution ophthalmic optical coherence tomographyò, Nat. Med. 7, 502ï 507, 2001.

[2] A. F. Fercher, Optical Coherence Tomography: Technology and Applications (Springer, New York), chap. 4, 2008.

[3] P. Puvanathasan and K. Bizheva, ñSpeckle noise reduction algorithm for optical coherence tomography based on interval type II fuzzy setò, Opt. Express 15, 15747ï 15758, 2007.

[4] H. Salinas and D. Fernández, ñComparison of pde-based nonlinear diffusion approaches for image enhancement and denoising in optical coherence tomographyò, IEEE Trans. Med. Imaging 26, 761ї 771, 2007.

[5] G. Gilboa, N. Sochen and Y. Zeevi, ñImage enhancement and denoising by complex diffusion processò, IEEE Trans. Pattern Anal. Mach. Intell., 26, 1020ї 1036, 2004.

[6] R.J. Zawadzki, A.R. Fuller, D.F. Wiley, B. Hamann, S.S. Choi, J.S. Werner, ñAdaptation of a support vector machine algorithm for segmentation and visualization of retinal structures in volumetric optical coherence tomography data setsò, J. Biomed. Optics, SPIE, 12, 041206, 2007.

[7] A. Fuller, R. Zawadzki, S. Choi, D. Wiley, J. Werner, B. Hamann, ñSegmentation of Three-dimensional Retinal Image Dataò, IEEE Transactions on Visualization and Computer Graphics, 13, 1719 -1726, 2007.

[8] R. Bernardes, C. Maduro, P. Serranho, A. Araújo, S. Barbeiro, and J. Cunha-Vaz, ñImproved adaptive complex diffusion despeckling filterò, Opt. Express 18, pp. 24048-24059, 2010.

[9] A. Araújo, S. Barbeiro and P. Serranho, ñStability of finite difference schemes for complex diffusion processesò, DMUC report 10-23, 2010.

[10] A. Bagci, R. Ansari, M. Shahidi, ñA method for detection of retinal layers by optical coherence tomography image segmentationò, Life Science Systems and Applications Workshop, 2007. LISA 2007. IEEE/NIH, 144 -147, 2007.

[11] D.C. Fernández, H. M. Salinas, C. A. Puliafito, Automated detection of retinal layer structures on optical coherence tomography images, Opt. Express, OSA, 13, 10200-10216, 2005. 